\title{
Integrability and linearizability for Lotka-Volterra systems with the $3:-q$ resonant saddle point
}

Qinlong Wang ${ }^{1,2^{*}}$ and Wentao Huang ${ }^{1,2}$

\section{"Correspondence:}

wqinlong@163.com

'School of Science, Hezhou

University, Hezhou, 542800,

P.R. China

${ }^{2}$ Guangxi Key Laboratory of Trusted

Software, Guilin University of Electronic Technology, Guilin 541004, P.R. China

\begin{abstract}
Integrability and linearizability of a Lotka-Volterra system in a neighborhood of the singular point with eigenvalues 3 and any negative integer $-q$ are studied completely. By computing the singular point quantities and generalized period constants, we obtain, respectively, the integrable and linearizable necessary conditions for this class of systems. Then we apply some effective ways to prove the sufficiency. Here the algorithms of finding necessary conditions are all linear and readily done using computer algebra system such as Mathematica or Maple, and these play an important role in solving completely the integrability and linearizability for the $3:-q$ resonant case.
\end{abstract}

MSC: $34 C x x$

Keywords: Lotka-Volterra system; integrability; linearizability; generalized center

\section{Introduction}

This paper is concerned with integrability and linearizability for the following systems with linear part of $p:-q$ resonant saddle point type:

$$
\frac{\mathrm{d} x}{\mathrm{~d} t}=p x+P(x, y), \quad \frac{\mathrm{d} y}{\mathrm{~d} t}=-q y+Q(x, y)
$$

where $p, q \in \mathbb{Z}^{+}, x, y, t \in \mathbb{R}, P$ and $Q$ are polynomials. After a time scaling $t \rightarrow p t$, system (1) can be also expressed into the following form:

$$
\frac{\mathrm{d} x}{\mathrm{~d} t}=x+P(x, y), \quad \frac{\mathrm{d} y}{\mathrm{~d} t}=-\lambda y+Q(x, y)
$$

where $\lambda=\frac{q}{p} \in \mathbb{Q}^{+}$. For the above systems, most of the known work was focused on some special resonant, yet for the general case there are few results. For the $1:-2$ resonant saddle point of quadratic systems, by computing the saddle numbers to get the necessary conditions, the authors completely solved the integrability problem in [1, 2], and furthermore in [3], mainly by annihilating the coefficients of the normal form and finding the Darboux factor, the authors obtained the necessary and sufficient conditions (15 cases) for the linearizable systems. For the $1:-n$ resonant cases, integrability and linearizability of Lotka-Volterra systems were solved, respectively, in [2] and [3]. As for the general case $2:-p$, the authors of [4] fully discussed the two problems by generalizing and expanding 
two methods already known: the power expansion of the first integral and the transformation of the saddle into a node. For the general case of the $p:-q$ resonance, the authors of [5] studied systematically the mechanisms which lead to the origin being linearizable, integrable or normalizable. Recently the authors in [6] gave the center variety for families of $p:-q$ resonant polynomial vector fields, and moreover derived an algorithm for computing the focus quantities. Especially there followed, for cubic Lotka-Volterra systems, some good results, obtained in [7-9]. However, one is far from finishing the investigation of all Lotka-Volterra systems.

Here we investigate completely Lotka-Volterra systems with any $3:-q$ resonance as follows:

$$
\begin{aligned}
& \dot{x}=x\left(3+a_{2} x+a_{1} y\right), \\
& \dot{y}=-y\left(q+b_{1} x+b_{2} y\right),
\end{aligned}
$$

where $q$ is an arbitrary positive integer, for which only several particular cases were considered in $[10,11]$. Based on the developed algorithm of a singular point quantity in [12, 13] and the generalized algorithm of period constants in [11], we find all integrable and linearizable necessary conditions. Furthermore we apply some effective ways mainly from the known results, for example, the theory of Darboux first integrals to show sufficiency. Thus we consider the following more general complex polynomial differential systems with a resonant singular point:

$$
\begin{aligned}
& \frac{\mathrm{d} z}{\mathrm{~d} T}=p z+\sum_{k=2}^{\infty} Z_{k}(z, w)=Z(z, w), \\
& \frac{\mathrm{d} w}{\mathrm{~d} T}=-q w-\sum_{k=2}^{\infty} W_{k}(z, w)=-W(z, w),
\end{aligned}
$$

and similarly, after a time scaling $T \rightarrow p T$, the above system (4) can be also expressed into the following form:

$$
\begin{aligned}
& \frac{\mathrm{d} z}{\mathrm{~d} T}=z+\sum_{k=2}^{\infty} Z_{k}(z, w)=Z(z, w), \\
& \frac{\mathrm{d} w}{\mathrm{~d} T}=-\lambda w-\sum_{k=2}^{\infty} W_{k}(z, w)=-W(z, w),
\end{aligned}
$$

where $z, w, T \in \mathbb{C}, p, q \in \mathbb{Z}^{+},(p, q)=1, \lambda=\frac{q}{p}$ and $Z_{k}(z, w), W_{k}(z, w)$ are homogeneous polynomials in $(z, w)$, i.e., $Z_{k}=\sum_{\alpha+\beta=k} a_{\alpha \beta} z^{\alpha} w^{\beta}, W_{k}=\sum_{\alpha+\beta=k} b_{\alpha \beta} w^{\alpha} z^{\beta}$.

Obviously, one can see that: (i) If $z, w, T \in \mathbb{R}$ and the coefficients are all real, we have system (4), that is, system (1). (ii) If $p=q=1$ and the coefficients of system (4) satisfy the conjugate condition, i.e.,

$$
\overline{a_{\alpha \beta}}=b_{\alpha \beta}, \quad \alpha \geq 0, \beta \geq 0, \alpha+\beta \geq 2,
$$

then by means of the transformation

$$
z=x+y \mathbf{i}, \quad w=x-y \mathbf{i}, \quad T=\mathbf{i} t, \quad \mathbf{i}=\sqrt{-1},
$$


system (4) can be transformed into its concomitant system, i.e., the real planar differential system

$$
\frac{\mathrm{d} x}{\mathrm{~d} t}=-y+P(x, y), \quad \frac{\mathrm{d} y}{\mathrm{~d} t}=x+Q(x, y)
$$

where $x, y, t \in \mathbb{R}, P$ and $Q$ are polynomials. For system (7), many results concerning centers and isochronous centers can be found in [14-19].

Let us first recall some basic definitions and results (also see $[11,20]$ ).

Lemma 1.1 System (4) can be reduced to the normal form

$$
\frac{\mathrm{d} \xi}{\mathrm{d} T}=p \xi\left(1+\sum_{i=1}^{\infty} p_{i} U^{i}\right), \quad \frac{\mathrm{d} \eta}{\mathrm{d} T}=-q \eta\left(1+\sum_{j=1}^{\infty} q_{i} U^{i}\right)
$$

where $U=\xi^{q} \eta^{p}$, by using a formal change of variables

$$
\xi=z+\sum_{k+j=2}^{\infty} A_{k j} z^{k} w^{j}, \quad \eta=w+\sum_{k+j=2}^{\infty} B_{k j} w^{k} z^{j}
$$

where $A_{q i+1, p i}=B_{p i+1, q i}=0, i=1,2, \ldots, p_{0}=q_{0}=1, p_{i}$ and $q_{i}$ are polynomials of $a_{\alpha \beta}, b_{\alpha \beta}$ with rational coefficients, and $A_{k j}, B_{k j}$ can be determined uniquely, for $i, j=1,2,3, \ldots$.

We write $\mu_{0}=\tau_{0}=0, \mu_{k}=p_{k}-q_{k}, \tau_{k}=p p_{k}+q q_{k}, k=1,2,3, \ldots$

Definition 1.1 For any positive integer $k, \mu_{k}$ is called the $k$ th singular point quantity of the origin of system (4), and the origin of system (4) is called the generalized center, i.e., system (4) is integrable at the origin if $\mu_{k}=0$, i.e., $p_{k}=q_{k}, k=1,2,3, \ldots$.

Definition 1.2 For any positive integer $k, \tau_{k}$ are called the $k$ th generalized period constants of the origin of system (4). And system (4) can be called linearizable at the origin if $\tau_{k}=\mu_{k}=0$, i.e., $p_{k}=q_{k}=0, k=1,2,3, \ldots$.

Lemma $1.2([1,4])$ System (4) is linearizable at the origin if it is integrable at the origin and there exists an analytic change of variables (9), such that one of the two equations $\dot{\xi}=p \xi$ and $\dot{\eta}=-q \eta$ holds.

In the next section, we deduce a recursive formula to compute the singular point quantities of system (3) and the integrable necessary conditions for system (3) are obtained. In Section 3, we deduce also a recursive formula to compute generalized period constants of the systems, and at the same time, all linearizable necessary conditions of the origin for system (3) are obtained. In Section 4, the proofs of sufficient conditions are given completely.

\section{Generalized center at the origin}

First we discuss computational method of singular point quantities for system (3). 
Lemma $2.1([12,13])$ For system (4), using the program of term by term calculations, we can determine a formal power series,

$$
F(z, w)=z^{q} w^{p}+\sum_{\alpha+\beta=p+q+1}^{\infty} c_{\alpha \beta} z^{\alpha} w^{\beta}
$$

where $c_{q p}=1, c_{m q, m p}=0$ for $m=2,3, \ldots$, such that

$$
\left.\frac{\mathrm{d} F}{\mathrm{~d} T}\right|_{(4)}=\sum_{m=1}^{\infty} \lambda_{m}\left(z^{q} w^{p}\right)^{m+1}
$$

The relations between $\mu_{i}=p_{i}-q_{i}$ and $\lambda_{i}(i=1,2, \ldots)$ are as follows.

Lemma 2.2 Let $p_{0}=q_{0}=1, \mu_{0}=\lambda_{0}=0$. If a positive integer l exists, such that

$$
p_{0}=q_{0}, \quad p_{1}=q_{1}, \quad \ldots, \quad p_{l-1}=q_{l-1}, \quad \text { i.e. } \quad \mu_{0}=\mu_{1}=\cdots=\mu_{l-1}=0,
$$

then

$$
\lambda_{0}=\lambda_{1}=\cdots=\lambda_{l-1}=0, \quad \lambda_{l}=p q \mu_{l} .
$$

The converse case holds as well.

Remark 1 Similar to the proof procedure in $[11,15]$, we can obtain the above relation, thus $\lambda_{l}$ and $p q \mu_{l}$ are called algebraic equivalent, i.e., $\lambda_{l} \sim p q \mu_{l}[14,15]$. Further, if $z, w, T \in \mathbb{R}$ and the coefficients of system (4) are all real, namely it is just system (1), for the $p:-q$ resonant focus number $g_{k}$ in [2], then $g_{k}=\lambda_{k}, k=1,2, \ldots$. So we can apply directly the above method to find the necessary conditions of integrability for solving the problem of the generalized center.

Now we consider the real system (3).

Theorem 2.3 For system (3), using the program of term by term calculations, we can determine a formal power series,

$$
H(x, y)=x^{q} y^{3}+\sum_{\alpha+\beta=q+4}^{\infty} c_{\alpha \beta} x^{\alpha} y^{\beta}
$$

such that

$$
\left.\frac{\mathrm{d} H}{\mathrm{~d} t}\right|_{(3)}=\sum_{m=1}^{\infty} \lambda_{m}\left(x^{q} y^{3}\right)^{m+1}
$$

where $c_{q, 3}=1, c_{m q, 3 m}=0$ for $m=2,3, \ldots$, then $\forall(\alpha, \beta)$, when $3 \alpha \neq q \beta(i . e .,|\alpha-(m+1) q|+\mid \beta-$ $3(m+1) \mid \neq 0, m=1,2, \ldots), c_{\alpha \beta}$ is determined uniquely by the following recursive formula:

$$
c_{\alpha \beta}=-\frac{1}{3 \alpha-q \beta}\left[\left((\alpha-1) a_{2}-\beta b_{1}\right) c_{\alpha-1, \beta}+\left(\alpha a_{1}-(\beta-1) b_{2}\right) c_{\alpha, \beta-1}\right]
$$


and for any positive integer $m, \lambda_{m}$ is determined uniquely by the following recursive formula:

$$
\begin{aligned}
\lambda_{m}= & {\left[(m q+q-1) a_{2}-3(m+1) b_{1}\right] c_{m q+q-1,3 m+3} } \\
& +\left[(m+1) q a_{1}-(3 m+2) b_{2}\right] c_{m q+q, 3 m+2} ;
\end{aligned}
$$

in the above expressions, if $\alpha<0$ or $\beta<0$, let $c_{\alpha \beta}=0$, and $\lambda_{m}$ is the mth singular point quantity of the origin of system (3).

First we verify the integrability for Lotka-Volterra systems with the $3:-1$ and $3:-2$ resonances, which have been studied in $[1,4]$, respectively.

Corollary 2.4 For $q=1,2$, the origin of system (3) is a generalized center if and only if the following conditions are satisfied:

when $q=1$,

$$
\lambda_{1}=-\frac{9}{2} a_{1}\left(a_{1}-b_{2}\right)\left(a_{1} a_{2}-2 a_{2} b_{2}-3 b_{1} b_{2}\right)=0,
$$

when $q=2$,

$$
\lambda_{1}=\frac{27}{8} a_{1} b_{1}\left(2 a_{1}+b_{2}\right)\left(2 a_{1} a_{2}-a_{2} b_{2}-3 b_{1} b_{2}\right)=0 .
$$

Proof By applying the recursive formulas, one can obtain the necessary conditions (18) and (19). It is easily verified that the above conditions are identical to the corresponding results in $[1,4]$. The proof of these sufficient conditions will not be given any longer.

Remark 2 For system (3), according to Theorem 2.3, we can get the recursive formulas to compute the singular point quantities for investigating the generalized center for any positive integer $q$. The integrability and linearizability for Lotka-Volterra systems with $1:-n$ or $n:-1, n \in \mathbb{N}$, resonances have been studied in [1] completely, so we only need to consider the case of $q=3 K+1,3 K+2(K=1,2, \ldots)$.

For system (3), we can compute the singular point quantities and obtain the following result.

Theorem 2.5 The first 20 singular point quantities for the origin of system (3) are as follows:

when $q=3 K+1, K=1,2, \ldots$,

$$
\begin{aligned}
& \lambda_{1}=a_{1} f_{q} f_{1 K} \prod_{i=0}^{K-1}\left[(3 i-2) a_{2}-3 b_{1}\right] \prod_{i=0}^{K-1}\left[(3 i) a_{2}-3 b_{1}\right], \\
& \lambda_{2}=a_{1} f_{q} f_{3 K} \prod_{i=0}^{K-1}\left[(3 i-2) a_{2}-3 b_{1}\right] \prod_{i=0}^{K-1}\left[(3 i) a_{2}-3 b_{1}\right], \\
& \lambda_{3}=\lambda_{4}=\cdots=\lambda_{20}=0,
\end{aligned}
$$


when $q=3 K+2, K=1,2, \ldots$

$$
\begin{aligned}
& \lambda_{1}=a_{1} f_{q} f_{2 K} \prod_{i=0}^{K-1}\left[(3 i-1) a_{2}-3 b_{1}\right] \prod_{i=0}^{K}\left[(3 i) a_{2}-3 b_{1}\right], \\
& \lambda_{2}=a_{1} f_{q} f_{4 K} \prod_{i=0}^{K-1}\left[(3 i-1) a_{2}-3 b_{1}\right] \prod_{i=0}^{K}\left[(3 i) a_{2}-3 b_{1}\right], \\
& \lambda_{3}=\lambda_{4}=\cdots=\lambda_{20}=0,
\end{aligned}
$$

where

$$
f_{q}=q a_{1} a_{2}+(q-3) a_{2} b_{2}-3 b_{1} b_{2}
$$

and $f_{1 K}, f_{2 K}, f_{3 K}, f_{4 K}$ are polynomials in $a_{1}, a_{2}, b_{1}, b_{2}$ with the form as follows, respectively:

$$
\begin{aligned}
& f_{1 K}, f_{2 K} \triangleq \sum_{i=0}^{K} a_{2}^{i} b_{1}^{K-i}\left(\alpha_{i} a_{1}+\beta_{i} b_{2}\right), \\
& f_{3 K}, f_{4 K} \triangleq \sum_{i=0}^{K+q} a_{2}^{i} b_{1}^{K+q-i}\left(\gamma_{i 0} a_{1}^{4}+\gamma_{i 1} a_{1}^{3} b_{2}+\gamma_{i 2} a_{1}^{2} b_{2}^{2}+\gamma_{i 3} a_{1}^{1} b_{2}^{3}+\gamma_{i 4} b_{2}^{4}\right),
\end{aligned}
$$

where $\alpha_{i}, \beta_{i}, \gamma_{i j} \in \mathbb{R}, f_{1 K}$ and $f_{2 K}$ are different for different $\alpha_{i}, \beta_{i}$, similarly $f_{3 K}$ and $f_{4 K}$ for $\gamma_{i j}$. In the above expression of $\lambda_{l}$, we have already let $\lambda_{1}=\lambda_{2}=\cdots=\lambda_{l-1}=0, l=2,3, \ldots, 20$.

Then we have the following.

Theorem 2.6 (A) When $q=3 K+1, K=1,2, \ldots$, the origin of system (3) is a generalized center if and only if one of the following conditions is satisfied:

(i) $a_{1}=0$,

(ii) $\prod_{i=0}^{K-1}\left[(3 i-2) a_{2}-3 b_{1}\right]=0$,

(iii) $\prod_{i=0}^{K-1}\left[(3 i) a_{2}-3 b_{1}\right]=0$,

(iv) $f_{q}=q a_{1} a_{2}+(q-3) a_{2} b_{2}-3 b_{1} b_{2}=0$,

(v) $f_{1 K}=0, \quad f_{3 K}=0$.

(B) When $q=3 K+2, K=1,2, \ldots$, the origin of system (3) is a generalized center if and only if one of the following conditions is satisfied:
(I) $a_{1}=0$,
(II) $\prod_{i=0}^{K-1}\left[(3 i-1) a_{2}-3 b_{1}\right]=0$, 


$$
\begin{aligned}
& \text { (III) } \prod_{i=0}^{K}\left[(3 i) a_{2}-3 b_{1}\right]=0, \\
& \text { (IV) } f_{q}=q a_{1} a_{2}+(q-3) a_{2} b_{2}-3 b_{1} b_{2}=0, \\
& \text { (V) } f_{2 K}=0, \quad f_{4 K}=0 .
\end{aligned}
$$

Proof We have to find necessary conditions of the generalized center from the vanishing of all first singular point quantities. We can let the first 20 singular point quantities in equation (20) or (21) be zero. It is easy to obtain the above five necessary conditions.

Next, the sufficient conditions need to be proved. On the one hand, for the conditions (22), (23), (24) and (27), (28), (29) will be proved sufficiency, respectively, in Section 4. And furthermore, from Lemma 2.7, the condition (25) or (30) is sufficient.

On the other hand, from equation (26), i.e., $f_{1 K}=f_{3 K}=0$, when $a_{2} b_{2}=0$ holds, we can get the two conditions

$$
b_{2}=a_{1}=0 \quad \text { or } \quad a_{2}=b_{1}=0 \text {, }
$$

however, the two conditions are included in condition (22) and (23), (24), respectively. When $a_{2} b_{2} \neq 0$ holds, from $f_{1 K}=f_{3 K}=0$, we can have the two constants $r$ and $s$ such that $b_{1}=r a_{2}$ and $a_{1}=s b_{2}$ hold. And excluding the conditions which have been listed in equations (22), (23), (24), and (25), we can get the only three groups of conditions

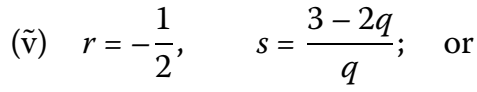

$$
\begin{aligned}
& r=-\frac{5}{3}, \quad s=1 ; \quad \text { or } \quad r=K, \quad s=\frac{4}{3 q+1}
\end{aligned}
$$

where $q=3 K+1, K=1,2, \ldots$.

By a similar discussion, from $f_{2 K}=f_{4 K}=0$, we can get the only two groups of conditions

$$
\text { (I) } \quad r=-\frac{1}{2}, \quad s=\frac{3-2 q}{q} ; \quad \text { or } \quad r=-\frac{4}{3}, \quad s=-\frac{1}{2}
$$

where $q=3 K+2, K=1,2, \ldots$.

However, the conditions $(\tilde{v})$ and $(\tilde{V})$ will be proved sufficient, respectively, in Section 4.

Lemma $2.7\left([4]\right.$, Theorem C) System (3) is integrable if $q a_{2}\left(a_{1}+b_{2}\right)=3 b_{2}\left(a_{2}+b_{1}\right)$.

\section{Linearizability at the origin}

Now, we discuss generalized isochronicity of the origin for system (3), namely we figure out all linearizable conditions of the system. First we introduce the algorithm of computing the generalized period constants $\tau_{i}=p p_{i}+q q_{i}, i=1,2, \ldots$, which has been given in [11].

Theorem 3.1 For system (3), we can derive uniquely the following formal series:

$$
f(x, y)=x+\sum_{k+j=2}^{\infty} c_{k j} x^{k} y^{j}, \quad g(x, y)=y+\sum_{k+j=2}^{\infty} d_{k, j} y^{k} x^{j},
$$


where $c_{q i+1,3 i}=d_{3 i+1, q i}=0, i=1,2, \ldots$, such that

$$
\frac{\mathrm{d} f}{\mathrm{~d} T}=3 f(x, y)+\sum_{i=1}^{\infty} 3 p_{i}^{\prime} x^{q i+1} y^{3 i}, \quad \frac{\mathrm{d} g}{\mathrm{~d} T}=-q g(x, y)-\sum_{i=1}^{\infty} q q_{i}^{\prime} y^{3 i+1} x^{q i}
$$

and when $3-3 k+j q \neq 0$ (i.e., $|k-q i-1|+|j-3 i| \neq 0, i=0,1,2, \ldots), c_{k j}$ is determined by the following recursive formula:

$$
c_{k j}=\frac{1}{3-3 k+j q}\left[\left((k-1) a_{2}-j b_{1}\right) c_{k-1, j}+\left(k a_{1}-(j-1) b_{2}\right) c_{k, j-1}\right],
$$

and when $3 j+q-k q \neq 0$ (i.e., $|k-3 i-1|+|j-q i| \neq 0, i=0,1,2, \ldots), d_{k j}$ is determined by the following recursive formula:

$$
d_{k j}=\frac{1}{3 j+q-k q}\left[\left((k-1) b_{2}-j a_{1}\right) d_{k-1, j}+\left(k b_{1}-(j-1) a_{2}\right) d_{k, j-1}\right],
$$

and for any positive integer $i, p_{i}^{\prime}$ and $q_{i}^{\prime}$ are determined by the following recursive formulas:

$$
\begin{aligned}
& p_{i}^{\prime}=i\left(q a_{2}-3 b_{1}\right) c_{i q, 3 i}+\left[(i q+1) a_{1}-(3 i-1) b_{2}\right] c_{i q+1,3 i-1}, \\
& q_{i}^{\prime}=i\left(3 b_{2}-q a_{1}\right) d_{3 i, i q}+\left[(3 i+1) b_{1}-(i q-1) a_{2}\right] d_{3 i+1, i q-1} .
\end{aligned}
$$

In the expressions (36), (37), (38), and (39), we have let $c_{1,0}=d_{1,0}=1, c_{0,1}=d_{0,1}=0$, and if $\alpha<0$ or $\beta<0$, we let $a_{\alpha \beta}=b_{\alpha \beta}=c_{\alpha \beta}=d_{\alpha \beta}=0$.

The relations between $p_{i}, q_{i}$ and $p_{i}^{\prime}, q_{i}^{\prime}(i=1,2, \ldots)$ are as follows.

Theorem 3.2 Let $p_{0}=q_{0}=p_{0}^{\prime}=q_{0}^{\prime}=0$. If a positive integer l exists, such that

$$
p_{0}=q_{0}=p_{1}=q_{1}=\cdots=p_{l-1}=q_{l-1}=0,
$$

then

$$
p_{0}^{\prime}=q_{0}^{\prime}=p_{1}^{\prime}=q_{1}^{\prime}=\cdots=p_{l-1}^{\prime}=q_{l-1}^{\prime}=0, \quad p_{l}=p_{l}^{\prime}, q_{l}=q_{l}^{\prime} .
$$

The converse also holds true.

The algorithm of Theorem 3.1 and Theorem 3.2 gives a method to determine linearizable systems and find the necessary conditions for system (3). By applying the recursive formulas, the authors of [11] verify the linearizable conditions for Lotka-Volterra systems with the $3:-1$ and $3:-2$ resonance, which have been studied in $[1,4]$, respectively.

Now we consider the linearizability by investigating the two former cases of generalized center conditions, respectively, for $q=3 K+1$ or $3 K+2, K=1,2, \ldots$.

Case (a): Consider the generalized center condition in one the of Cases (i), (ii), (iii), ( $\tilde{\mathrm{v}}$ ), and (I), (II), (III), (V). In fact, for the case of $q=3 K+1$, if equation (22) or (23) or (24) or (32) holds, putting expression into the recursive formulas in Theorem 3.1, respectively, we can always obtain the first pairs of coefficients of the normal form for the saddle point are zero, for example

$$
p_{1}=q_{1}=p_{2}=q_{2}=\cdots=p_{20}=q_{20}=0,
$$


at the same time, for the case of $q=3 K+2$, if equation (27) or (28) or (29) or (33) holds, there exist similar results. Therefore the above ones are judged as linearizable conditions at the origin.

Case (b): Consider the generalized center conditions in Case (iv) and (IV). When (iv) or (IV) holds, obviously we may consider only the case of $a_{2} b_{2} \neq 0$, from $f_{q}=q a_{2}\left(a_{1}+b_{2}\right)-$ $3 b_{2}\left(b_{1}+a_{2}\right)=0$, we can also have two constants $r$ and $s$ such that $b_{1}=r a_{2}$ and $a_{1}=s b_{2}$ hold. And furthermore from equation (25) or (30), we can get the only condition, $s=\frac{3+3 r-q}{q}$, and then putting it into the recursive formulas in Theorem 3.1, from Theorem 3.2 we have the first 20 pairs of coefficients of the normal form for the saddle point, which are as follows:

when $q=3 K+1$,

$$
\begin{aligned}
& p_{1}=q_{1}=\frac{1}{2 q^{3} 3^{2 K} q !} a_{2}^{q} b_{2}^{3} \prod_{i=-3}^{q-1}(i-3 r), \\
& p_{2}=q_{2}=\cdots=p_{20}=q_{20}=0,
\end{aligned}
$$

when $q=3 K+2$,

$$
\begin{aligned}
& p_{1}=q_{1}=\frac{1}{2 q^{3} 3^{2 K+1} q !} a_{2}^{q} b_{2}^{3} \prod_{i=-3}^{q-1}(i-3 r) \\
& p_{2}=q_{2}=\cdots=p_{20}=q_{20}=0
\end{aligned}
$$

Obviously, all the above coefficients vanish if and only if $\prod_{i=-3}^{q-1}(i-3 r)=0$. Then we can get the conditions

$$
r=\frac{i}{3}, \quad s=\frac{3+3 r-q}{q}, \quad i=-3,-2,-1,0,1,2, \ldots, q-1 .
$$

However, some conditions are included in condition (22), (23) or (27), (28), excluding the conditions, so we can get the only following conditions:

when $q=3 K+1$,

$$
r=\frac{i}{3}, \quad s=\frac{3+3 r-q}{q}, \quad i=-3, \underbrace{-1,2,5, \ldots, q-2}_{3 j-1, j=0,1, \ldots, K}, q-1
$$

when $q=3 K+2$,

$$
r=\frac{i}{3}, \quad s=\frac{3+3 r-q}{q}, \quad i=-3, \underbrace{-2,1,4, \ldots, q-4, q-1}_{3 j-2, j=0,1, \ldots, K+1} .
$$

Theorem 3.3 For the origin of system (3):

(A) when $q=3 K+1(K=1,2, \ldots)$, it is linearizable at the origin if and only if one of the conditions (22) (23), (24), (32), and (45) is satisfied;

(B) when $q=3 K+2(K=1,2, \ldots)$, it is linearizable at the origin if and only if one of the conditions (27) (28), (29), (33), and (46) is satisfied.

The necessary conditions of Theorem 3.3 are obvious, the proof of sufficient conditions will be given in Section 4 . 


\section{The proof of sufficient conditions}

In the process of proving the sufficient conditions in Theorems 2.6 and 3.3, we will apply several well-known results.

Lemma 4.1 ([3]) The real system

$$
\dot{x}=x+c_{20} x^{2}+c_{11} x y+c_{02} y^{2}, \quad \dot{y}=-\lambda y+d_{02} y^{2}
$$

for $\lambda$ is always linearizable if $1 / \lambda \notin \mathbb{N}$.

From $a_{1}=0$ in condition (i) or (I), we can apply Lemma 4.1, so system (3) is linearizable and also integrable.

Lemma 4.2 ([4], Theorem D) For the real system (3) with $\lambda=\frac{q}{3} \in \mathbb{Q}^{+}$and $(q, 3)=1$, if $\lambda \in \mathbb{Q}$ and $q / 3-\frac{b_{1}}{a_{2}}=n \in \mathbb{N}, 2 \leq n<q / 3+1$ hold, for arbitrary parameter $\lambda$, it is linearizable at the origin.

If the condition (ii) or (II), namely equation (23) or (28) holds, we can apply Lemma 4.2, thus it is linearizable at the origin of system (3).

Lemma 4.3 ([4], Theorem E) For the real system (3) with $\lambda=\frac{q}{3}>1$ and $\frac{b_{1}}{a_{2}}=r \in \mathbb{N}^{*}$, it is linearizable at the origin if one of the following conditions holds:

$$
\begin{aligned}
& \left(\mathrm{E}_{1}\right) \quad \lambda \in \mathrm{R} \backslash \mathrm{Q}, \quad 1 \leq r<q / 3, \\
& \left(\mathrm{E}_{2}\right) \quad \lambda \in \mathrm{Q}, \quad 1 \leq r<q / 3-1, \\
& \left(\mathrm{E}_{3}\right) \quad \lambda \in \mathrm{Q}, \quad q / 3-1<r<q / 3, \quad q / 3 \neq r+\frac{1}{3}, \\
& \left(\mathrm{E}_{4}\right) \quad \lambda=\frac{q}{3}=r+\frac{1}{3}, \quad \frac{b_{2}}{a_{1}}=\frac{q}{2} .
\end{aligned}
$$

If the condition (iii) or (III), namely equation (24) or (29) holds, we can apply $\left(\mathrm{E}_{2}\right)$ and $\left(\mathrm{E}_{3}\right)$ in Lemma 4.3 except for the case of $b_{1}=0$, however, for the case of $b_{1}=0$, we can also apply Lemma 4.1. Therefore, under the condition (iii) or (III), it is linearizable at the origin of system (3).

Furthermore, we have the following.

Theorem 4.4 If $b_{2}=-a_{1}, b_{1}=-a_{2}$ holds, there exists a change of coordinates which transforms system (3) into $\dot{u}=3 u, \dot{v}=-q v$, namely,

$$
\begin{aligned}
& u=x\left(3 q-3 a_{1} y+q a_{2} x\right)^{-1}, \\
& v=y\left(3 q-3 a_{1} y+q a_{2} x\right)^{-1}
\end{aligned}
$$

thus system (3) is linearizable.

Theorem 4.5 For system (3), if $b_{1}=r a_{2}, a_{1}=s b_{2}, r=(3 j-1) / 3, q=3 K+1, s=(3 r-q+3) / q$, $j=0,1,2, \ldots, K, K=1,2, \ldots$ hold, it is linearizable at the origin. 
Proof When the above conditions hold, system (3) is of the following form:

$$
\begin{aligned}
& \dot{x}=3 x+\frac{1-3 K+3 j}{1+3 K} b_{2} x y+a_{2} x^{2}, \\
& \dot{y}=-(1+3 K) y-\frac{1}{3} a_{2}(-1+3 j) x y-b_{2} y^{2} .
\end{aligned}
$$

And there exists a linear change of coordinates: $(u, v)=\left(1+\frac{1}{3} a_{2} x+\frac{1}{1+3 K} b_{2} y, y\right)$, which transforms system (48) into the following form:

$$
\begin{aligned}
& \dot{u}=-3 u-\frac{4+3 K}{1+3 K} b_{2} u v+3 u^{2}, \\
& \dot{v}=-(2+3 K-3 j) v+(1-3 j) u v-\frac{2+3 K-3 j}{1+3 K} b_{2} v^{2} .
\end{aligned}
$$

We note that the origin is sent to a node and

$$
\Lambda=\frac{2+3 K-3 j}{3} \notin \mathbb{N}
$$

which ensures that the node of system (49) is linearizable by an analytic change of coordinates (the Poincaré theorem, also see [3]), in fact, there exist

$$
U(u, v)=u+\sum_{\alpha+\beta=2}^{\infty} a_{\alpha \beta} u^{\alpha} v^{\beta}, \quad V(u, v)=v+\sum_{\alpha+\beta=2}^{\infty} b_{\alpha \beta} v^{\alpha} u^{\beta}
$$

such that $\dot{U}=-3 U$ and $\dot{V}=-(2+3 K-3 j) V$, and we find

$$
\xi=\frac{x}{U}, \quad \eta=V U^{(3 j-1) / 3}
$$

such that $\dot{\xi}=3 \xi$ and $\dot{\eta}=-(3 K+1) \eta$; system (48) is therefore linearizable at the origin.

Theorem 4.6 For system (3), if $b_{1}=r a_{2}, a_{1}=s b_{2}, r=(3 j-2) / 3, q=3 K+2, s=(3 r-q+3) / q$, $j=0,1,2, \ldots, K, K=1,2, \ldots$ hold, it is linearizable at the origin.

Proof When the above conditions hold, system (3) is of the following form:

$$
\begin{aligned}
& \dot{x}=3 x-\frac{1+3 K-3 j}{2+3 K} b_{2} x y+a_{2} x^{2}, \\
& \dot{y}=-(2+3 K) y-b_{2} y^{2}+\frac{(2-3 j)}{3} a_{2} x y .
\end{aligned}
$$

And there exists a linear change of coordinates: $(u, v)=\left(1+\frac{1}{3} a_{2} x+\frac{1}{2+3 K} b_{2} y, y\right)$, which transforms system (51) into the following form:

$$
\begin{aligned}
& \dot{u}=-3 u-\frac{5+3 K}{2+3 K} b_{2} u v+3 u^{2}, \\
& \dot{v}=-(4+3 K-3 j) v+(2-3 j) u v-\frac{4+3 K-3 j}{2+3 K} b_{2} v^{2} .
\end{aligned}
$$


We note that the origin is sent to a node and

$$
\Lambda=\frac{4+3 K-3 j}{3} \notin \mathbb{N},
$$

which ensures that the node of system (52) is linearizable, and similar to the proof procedure in Theorem 4.5, one can see that system (51) is also linearizable at the origin.

From Theorems 4.4, 4.5, 4.6 and $\left(\mathrm{E}_{4}\right)$ in Lemma 4.3, one can see that if condition (45) or (46) holds, it is linearizable at the origin of system (3).

Furthermore, we have the following.

Theorem 4.7 For system (3), if $b_{1}=r a_{2}, a_{1}=s b_{2}, r=-4 / 3, s=-1 / 2, q=3 K+2, K=1,2, \ldots$ hold, it is linearizable at the origin.

Proof When the above conditions hold, system (3) is of the following form:

$$
\begin{aligned}
& \dot{x}=3 x-\frac{1}{2} b_{2} x y+a_{2} x^{2}, \\
& \dot{y}=-(2+3 K) y+\frac{4}{3} a_{2} x y-b_{2} y^{2} .
\end{aligned}
$$

And there exists a linear change of coordinates: $(u, v)=\left(x, 1+\frac{8}{12-9 K} a_{2} x+\frac{1}{2+3 K} b_{2} y\right)$, which transforms system (53) into the following form:

$$
\begin{aligned}
& \dot{u}=\frac{(8+3 K)}{2} u-\frac{2+3 K}{2} u v+\frac{(20+3 K)}{3(4-3 K)} a_{2} u^{2}, \\
& \dot{v}=(2+3 K) v+\frac{8(5+3 K)}{3(4-3 K)} a_{2} u v-(2+3 K) v^{2}-\frac{8(4+3 K)}{3(4-3 K)^{2}} a_{2}^{2} u^{2} .
\end{aligned}
$$

We note that the origin is sent to a node and

$$
\Lambda=\frac{2(2+3 K)}{8+3 K} \notin \mathbb{N}
$$

which ensures that the node of system (54) is linearizable, and similar to the proof procedure in Theorem 4.5, one can see that system (53) is also linearizable at the origin.

Theorem 4.8 For system (3), if $b_{2}=\frac{4+9 K}{4} a_{1}, b_{1}=K a_{2}, q=3 K+1, K=1,2, \ldots$ hold, it is linearizable at the origin.

Proof When the above conditions hold, system (3) is of the following form:

$$
\begin{aligned}
& \dot{x}=3 x+\frac{4}{4+9 K} b_{2} x y+a_{2} x^{2}, \\
& \dot{y}=-(3 K+1) y-K a_{2} x y-b_{2} y^{2} .
\end{aligned}
$$

And there exists a linear change of coordinates: $(u, v)=\left(x, 1-\frac{K(4+9 K)}{4-6 K-27 K^{2}} a_{2} x+\frac{1}{1+3 K} b_{2} y\right)$, which transforms system (55) into the following form:

$$
\dot{u}=\frac{8+15 K}{4+9 K} u+\frac{4(1+3 K)}{4+9 K} u v+\frac{4-2 K-15 K^{2}}{4-6 K-27 K^{2}} a_{2} u^{2},
$$




$$
\begin{aligned}
\dot{v}= & (1+3 K) v-\frac{K(4+3 K)(4+9 K)}{4-6 K-27 K^{2}} a_{2} u v \\
& -\frac{2 K(2+3 K)(4+9 K)}{\left(4-6 K-27 K^{2}\right)^{2}} a_{2}^{2} u^{2}-(1+3 K) v^{2} .
\end{aligned}
$$

We note that the origin is sent to a node and

$$
\Lambda=\frac{(4+9 K)(1+3 K)}{8+15 K} \notin \mathbb{N},
$$

which ensures that the node of system (56) is linearizable, and similar to the proof procedure in Theorem 4.5, one can see that system (55) is also linearizable at the origin.

Theorem 4.9 If $q a_{1}=(3-2 q) b_{2}, 2 b_{1}=-a_{2}$ or $r=-1 / 2, s=(3-2 q) / q$ hold, there exist a first integral $M$ and a change of coordinates which transform system (3) into $\dot{v}=-q v$, namely,

$$
\begin{aligned}
& M=x^{\alpha} y^{-2}\left(3 q^{2}+a_{2} q^{2} x+6 b_{2} q y+3 b_{2}^{2} y^{2}\right)^{\beta}, \quad \alpha=-\frac{q+3}{3}, \beta=\frac{2 q-3}{6} \\
& \nu=\frac{y}{\sqrt{3 q^{2}+a_{2} q^{2} x+6 b_{2} q y+3 b_{2}^{2} y^{2}}}
\end{aligned}
$$

and moreover system (3) is linearizable.

Obviously, applying Lemma 1.2, we can prove the above theorem easily.

Theorem 4.10 For system (3), if $b_{1}=r a_{2}, a_{1}=s b_{2}, r=-5 / 3, q=3 K+1, s=1, K=1,2, \ldots$ hold, it is linearizable at the origin.

Proof When the above conditions hold, system (3) is of the following form:

$$
\begin{aligned}
& \dot{x}=x\left(3+a_{2} x+b_{2} y\right)=X, \\
& \dot{y}=-y\left(3 K+1-\frac{5}{3} a_{2} x+b_{2} y\right)=Y .
\end{aligned}
$$

For system (58), we find an analytic integral factor in a neighborhood of the origin,

$$
M(x, y)=x^{3 K} y^{2}+\sum_{i=0}^{\infty} u_{i}(x) y^{i}
$$

such that

$$
\frac{\partial M}{\partial x} X+\frac{\partial M}{\partial y} Y+\left(\frac{\partial X}{\partial x}+\frac{\partial Y}{\partial y}\right) M=0
$$

where $u_{0}(x)=u_{1}(x)=0$,

$$
\begin{aligned}
& u_{2}(x)=-\frac{x^{3 K}}{\left(3+a_{2} x\right)^{3 K+7}}\left[\left(3+a_{2} x\right)^{3 K+7}-3^{3 K+7}\right] \\
& u_{3}(x)=\frac{x^{3 K}}{\left(3+a_{2} x\right)^{4 K+9}} \sum_{i=0}^{K+1} a^{[i]} x^{i}
\end{aligned}
$$




$$
\begin{aligned}
u_{i}(x)= & x^{\frac{(1+i)(1+3 K)}{3}-1}\left(3+a_{2} x\right)^{-(K+2)(i+1)-1} \\
& \times b_{2}\left(\int x^{-\frac{(1+i)(1+3 K)}{3}}\left(3+a_{2} x\right)^{(1+i)(2+K)}\left(i u_{i-1}(x)-x u_{i-1}^{\prime}(x)\right) d x\right), \quad i=4,5, \ldots,
\end{aligned}
$$

where all $a^{[i]} \in \mathrm{R}$ and the integral constants are all set to zero, then we can prove by induction that

$$
u_{i}(x)=x^{3 K}\left(3+a_{2} x\right)^{-(K+2)(i+1)-1} \sum_{j=0}^{(K+1)(i-2)} A_{i j}\left(a_{2} x\right)^{j}, \quad A_{i j} \in \mathbb{R}, i=4,5, \ldots
$$

And moreover the system is integrable at the origin with the above integral factor $M$; then we have

$$
\xi=x^{-3 /(q+1)} y^{-7 /(q+1)} M^{4 /(q+1)}
$$

such that $\dot{\xi}=3 \xi$, and we can apply Lemma 1.2 , so the system is linearizable.

From Theorems 4.7, 4.8,4.9, and 4.10, one can see that if condition $(\tilde{\mathrm{V}})$ or $(\tilde{\mathrm{V}})$, namely equation (32) or (33) holds, it is linearizable at the origin of system (3).

Thus, the proofs of Theorems 2.6 and 3.3 have been completed.

Remark 3 For system (3), in fact, if $K=0$, namely the case of $q=1,2$, the conclusions of Theorems 2.6 and 3.3 still hold. Thus we solve completely the integrability and linearizability of system (3).

\section{Competing interests}

The authors declare that they have no competing interests.

\section{Authors' contributions}

QW completed the main study, obtained the results of this article and drafted the manuscript. WH checked the proofs and verified the calculation. Both authors read and approved the final manuscript.

\section{Acknowledgements}

This work was supported by the doctor's scientific research foundation of Hezhou University (No. HZUBS201302), Guangxi Key Laboratory of Trusted Software (No. kx201336) and the National Natural Science Foundation of China (Nos. 11261013, 11371373).

\section{Received: 25 June 2013 Accepted: 23 December 2013 Published: 16 Jan 2014}

\section{References}

1. Fronville, A, Sadovski, A, Zoladek, H: Solution of the $1:-2$ resonant centre problem in the quadratic case. Fundam. Math. 157, 191-207 (1998)

2. Zoladek, $\mathrm{H}$ : The problem of center for resonant singular points of polynomial vector fields. J. Differ. Equ. 137, 94-118 (1997)

3. Christopher, C, Mardesic, P, Rousseau, C: Normalizable, integrable, and linearizable saddle points for complex quadratic systems in $\mathbb{C}^{2}$. J. Dyn. Control Syst. 9, 311-363 (2003)

4. Gravel, S, Thibault, P: Integrability and linearizability of the Lotka-Volterra system with a saddle point with rational hyperbolicity ratio. J. Differ. Equ. 184, 20-47 (2002)

5. Christopher, C, Rousseau, C: Normalizable, integrable and linearizable saddle points in the Lotka-Volterra system. Qual. Theory Dyn. Syst. 5, 11-61 (2004)

6. Romanovski, VG, Shafer, DS: On the center problem for $p:-q$ resonant polynomial vector fields. Bull. Belg. Math. Soc. Simon Stevin 15, 871-887 (2008)

7. $\mathrm{Hu}, \mathrm{Z}$, Romanovski, VG, Shafer, DS: 1 : -3 Resonant centers on $\mathbb{C}^{2}$ with homogeneous cubic nonlinearities. Comput. Math. Appl. 56, 1927-1940 (2008)

8. Chen, $X$, Giné, J, Romanovski, VG, Shafer, DS: The 1:-q resonant center problem for certain cubic Lotka-Volterra systems. Appl. Math. Comput. 218, 11620-11633 (2012) 
9. Dolićanin, D, Giné, J, Oliveira, R, Romanovski, VG: The center problem for a 2:-3 resonant cubic Lotka-Volterra system. Appl. Math. Comput. 220, 12-19 (2013)

10. Liu, C, Chen, G, Li, C: Integrability and linearizability of the Lotka-Volterra systems. J. Differ. Equ. 198, 301-320 (2004)

11. Wang, Q, Liu, Y: Linearizability of the polynomial differential systems with a resonant singular point. Bull. Sci. Math. $132,97-111(2008)$

12. Xiao, P: Critical point quantities and integrability conditions for complex planar resonant polynomial differential systems. PhD thesis, Central South University, P.R. China (2005) (in Chinese)

13. Wang, Q, Huang, W: Linear recursion formulas of generalized focus quantities and applications. Appl. Math. Comput. 219, 5233-5240 (2013)

14. Liu, Y, Li, J: Theory of values of singular point in complex autonomous differential system. Sci. China Ser. A 33, 10-24 (1990)

15. Liu, Y, Li, J, Huang, W: Singular Point Values Center Problem and Bifurcations of Limit Cycles of Planar Vector Fields. Science Press, Beijing (2008)

16. Chavarriga, J, Giné, J, García, I: Isochronous centers of a linear center perturbed by fourth degree homogeneous polynomial. Bull. Sci. Math. 123, 77-96 (1999)

17. Christopher, CJ, Devlin, J: Isochronous centers in planar polynomial systems. SIAM J. Math. Anal. 28, 162-177 (1997)

18. Mardesic, P, Rousseau, C, Toni, B: Linearization of isochronous centers. J. Differ. Equ. 121, 67-108 (1995)

19. Liu, Y, Huang, W: A new method to determine isochronous center conditions for polynomial differential systems. Bull. Sci. Math. 127, 133-148 (2003)

20. Amelbkin, BB, Lukasevnky, HA, Catovcki, AN: Nonlinear Vibration. БГУ Lenin Publ., Moscow (1982) (in Russian)

10.1186/1687-1847-2014-23

Cite this article as: Wang and Huang: Integrability and linearizability for Lotka-Volterra systems with the $3:-q$ resonant saddle point. Advances in Difference Equations 2014, 2014:23

\section{Submit your manuscript to a SpringerOpen ${ }^{\circ}$ journal and benefit from:}

- Convenient online submission

- Rigorous peer review

- Immediate publication on acceptance

Open access: articles freely available online

- High visibility within the field

- Retaining the copyright to your article 